\title{
Two-color x-ray free-electron laser by photocathode laser emittance spoiler
}

\author{
Carlo Vicario $\odot,{ }^{1,}{ }^{*}$ Simona Bettoni $\odot,{ }^{1}$ Alberto Lutman, ${ }^{2}$ Andreas Dax, \\ Martin Huppert $\odot,{ }^{1}$ and Alexandre Trisorio $\odot^{1}$ \\ ${ }^{1}$ Paul Scherrer Institute, 5232 Villigen-PSI, Switzerland \\ ${ }^{2}$ SLAC National Accelerator Laboratory, Menlo Park, California, USA
}

(Received 18 February 2021; accepted 14 June 2021; published 28 June 2021)

\begin{abstract}
Multispectral x-ray pump-probe experiments call for synchronized two-color free-electron lasers (FEL). This mode often implies a laborious setup or an inefficient use of the undulator. We report on a simple and noninvasive approach tested at SwissFEL for a two-color x-ray delivering almost $60 \%$ of the pulse energy compared with a single color. In this new method, a ps UV pulse is overlapped to the photocathode drive laser increasing the beam emittance, which locally inhibits the FEL process. This scheme permits highstability in energy and spectrum and the control of the two-color duration and intensity ratio. It enables shot-to-shot switching between one and two-color FEL and, since not associate to beam losses, it is compatible with high repetition-rate and high average-power FELs.
\end{abstract}

DOI: 10.1103/PhysRevAccelBeams.24.060703

Several x-ray free electron lasers (FELs) are currently in operation around the world [1-6]. At these facilities, fs or even sub-fs pulse durations, wavelengths tunable down to $1 \AA$ and mJ-pulse energies can be achieved. The corresponding brilliance surpasses by several orders of magnitude that which is obtained at synchrotrons. Free-electron lasers offer x-rays with unprecedented peak power, temporal resolution and spatial coherence enabling advanced studies in biology, femtochemistry, material in extreme conditions and condensed matter physics including the production of exotic states of matter by nonlinear x-ray interactions [7]. For time-resolved studies, pump-probe experiments are generally implemented by combining a conventional laser pump with a $\mathrm{x}$-ray FEL probe. This technique enables monitoring the temporal evolution of a variety of photoinduced processes on an ultrafast timescale [8]. In this context, the reduction of the temporal jitter down to fs-level between the pump and the probe pulses is a persistent challenge [9]. X-ray pump, x-ray probe experiments employing a two-color FEL are expected to drastically reduce the temporal jitter while the two-wavelengths can be tuned to excite and to explore different resonances [10-12]. The time-delayed two-color output of hard x-ray FELs is well-suited for multidimensional Auger and transient grating spectroscopy, and for multicolor diffraction imaging $[10,13]$.

\footnotetext{
*carlo.vicario@psi.ch
}

Published by the American Physical Society under the terms of the Creative Commons Attribution 4.0 International license. Further distribution of this work must maintain attribution to the author(s) and the published article's title, journal citation, and DOI.
A two-color FEL output can be obtained by the uneven tuning of the undulator resonance or by the manipulation of the electron bunch properties. In the first approach, the undulator strength parameter $K$ of two sections are tuned to radiate at two wavelengths with the potential of independent control of the pointing and the polarization. The temporal separation of the two colors can be controlled with a dedicated magnetic chicane between the undulator modules $[14,15]$. A major drawback for this configuration is that the same electrons generate both colors, resulting in FEL operation far from saturation, with minimum nonzero delay between the pulses due to slippage effects. Two-color hard x-ray pulses having a maximum delay of $200 \mathrm{fs}$, a total pulse energy up to $30 \%$ with respect to the standard SASE and a relative wavelength separation of 30\% are reported in [15]. Alternatively, a fresh slice of the bunch can be set to lase in each undulator section, such that both the emission regions could reach saturation [16]. The fresh-slicing can be implemented with a time-dependent transverse kick [17], by transverse dispersion in the beam transport [18,19] or by transverse mismatch $[20,21]$. The same effect can be obtained by a two-wavelength laser seed, which is amplified in two undulators [22]. For two-color seeded FEL, tens of $\mu \mathrm{J}$ pulses, with $20 \%$ relative FEL photon energy offset and a maximum delay of 900 fs were demonstrated at wavelength of few tens of nm [22]. In general, fresh-slicing schemes allow large tunability and FEL saturation with up to $800 \mu \mathrm{J}$ around $700 \mathrm{eV}$ and a maximum delay between the two colors of $\approx 1$ ps [16]. However, fresh-slice schemes still require a short gain length which is challenging to be reached at the hard $\mathrm{x}$-ray regime and full saturation for the first color must be achieved upstream the chicane.

The two-color FELs by electron beam manipulation are achieved, with a dedicated complex machine setup, by twin 
bunches accelerated to different energy and thus lasing at two wavelengths $[23,24]$. The two bunches can be accelerated in the same radio frequency bucket (twin bunches) or in different ones. In the last case, it is also possible to tune the two FEL pulses at the same photon energy but their temporal delay is multiple of the radio frequency period (typically few hundreds of picoseconds). Energy exceeding $1 \mathrm{~mJ}$ corresponding to $\approx 30 \%$ of the normal self-amplified spontaneous emission (SASE) FEL is reported for tens of fs two-color twins pulses [24]. The transport of the electron beam sets the maximum relative color separation of $\approx 2 \%$ and the temporal delay below 200 fs [24]. To obtain two-color FEL with a single electron bunch it is possible to suppress the lasing from the central part, using two current spikes by wakefield with a dechirper [25] or a double-slotted foil within the beam as an emittance spoiler [26]. The beam manipulation methods have limited temporal and wavelength tunability and typically they require a time-consuming setup. Passive structures have been used to generate multipulses at $7.5 \mathrm{keV}$ having $\approx 40 \mu \mathrm{J}$ energy $(\approx 30 \%$ of the standard SASE), temporal separation of $200 \mathrm{fs}$ and $2 \%$ relative wavelength offset [25]. With a foil having two slots placed on the beam, few fs two-color FEL around $1 \AA$ was demonstrated. In this approach, the peak power exceeds $10 \mathrm{GW}$, while the temporal and wavelength separation between the two-color pulses is $\approx 150$ fs and $\approx 2 \%$ respectively [26]. Solid targets that spoil the electron bunch emittance present, however, the downside of significant radiation losses, preventing their applicability to high-repetition rate and high-average power x-ray FELs. A sextupole, in combination with a standard orbit control, was used to suppress the radiation from the bunch center, while keeping the head and the tail still lasing at two photon energies. With this method, two-color FEL around $7.2 \mathrm{keV}$, with energy $<100 \mu \mathrm{J}$, temporal and photon energy separation of up to 160 fs and $2 \%$ respectively was demonstrated [19]. Laser heater shaping [27] was demonstrated for single ten fs FEL pulses by spoiling the longitudinal emittance and was proposed for two-color FELs. This technique is compatible with high repetition rate accelerators, but introduces other issues due to the stability of the overlap between the laser and the electron beam. In general, all the above techniques require either precious beam time to implement a complicated electron beam shaping or an inefficient use of the undulator line, which eventually prevents the application of split-undulator schemes at harder x-rays.

This article reports on a new noninvasive and straightforward method for the generation of a two-color FEL by electron beam emittance spoiling based on the exploitation of two lasers at the photocathode. Different from other beam-based techniques, the presented method delivers two-color x-rays using the optimal single-color FEL settings without dedicated accelerator and undulator retuning thus saving beam-time. Moreover, it enables a lock-in mode (with shot-to-shot switching between two-color and single wavelength mode) and it is directly applicable to high-average power x-ray FELs because it is not contributing to beam losses. The concept is schematically depicted in Fig. 1. A short laser pulse (emittance spoiler) is overlapped to the nominal photocathode (PC) laser. The excess of charge leads, at low energy, to a localized increase of the emittance, which reduces and eventually prevents the FEL amplification. The part of the bunch not interacting with the laser spoiler still produces FEL emission which is centred at two wavelengths due to the linear energy chirp accumulated along the linear accelerator. This mechanism is particularly efficient for an optimized FEL where most of the electron bunch contributes to the $\mathrm{x}$-ray emission. The effect on the FEL process can be understood by referring to the condition on the transverse emittance $\epsilon$ that the beam should fulfil to assure that all the electrons radiate into the fundamental mode of the FEL [28]: $\epsilon / \gamma \leq \lambda / 4 \pi$, being $\gamma$ the relativistic Lorenz factor and $\lambda$ the FEL wavelength. At shorter $\lambda$, the condition on the emittance becomes more stringent and the FEL spectrum more sensitive to the laser emittance spoiler.

The time and wavelength separation between the two colors $(\Delta t$ and $\Delta \lambda)$ are key parameters for time-resolved experiments. These quantities may be tuned by changing the compression and the energy chirp of the electron beam. Using the low-energy compressor allows the control of $\Delta t$ with minor change on $\Delta \lambda$. The minimum $\Delta t$ corresponds to the overlap of the two colors while a maximum delay of few hundreds of fs still guarantees sufficient peak current and FEL amplification. $\Delta \lambda$ can be varied independently from

(a)
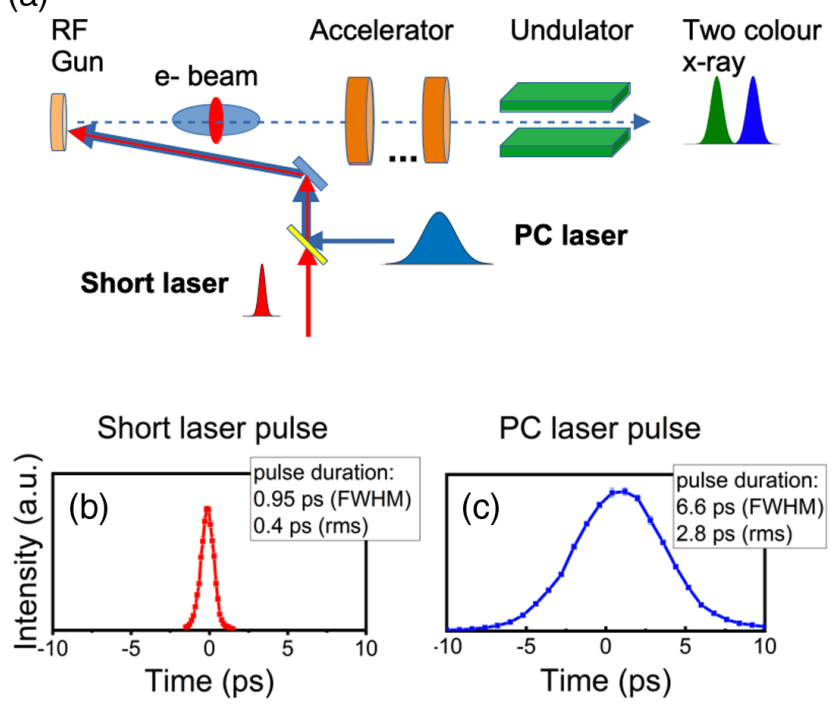

FIG. 1. (a) Proposed two-color FEL scheme. A ps long deepUV laser is overlapped to the nominal PC laser spoiling locally the emittance. The emittance increase is preserved through the accelerator and in the undulator producing two-color FEL corresponding to the low emittance portion of the beam. Optical cross-correlation traces for (b) emittance spoiler and (c) nominal photocathode lasers. 
TABLE I. Laser emittance spoiler and other two-color x-ray FEL methods demonstrated in literature. The top part of the table refers to the two-color FEL based on the manipulation of the electron beam while the bottom rows on the tuning of the undulator K. $\Delta \mathrm{t}$ and $\Delta \lambda$ are the delay between the two-color pulses and their wavelength separation respectively.

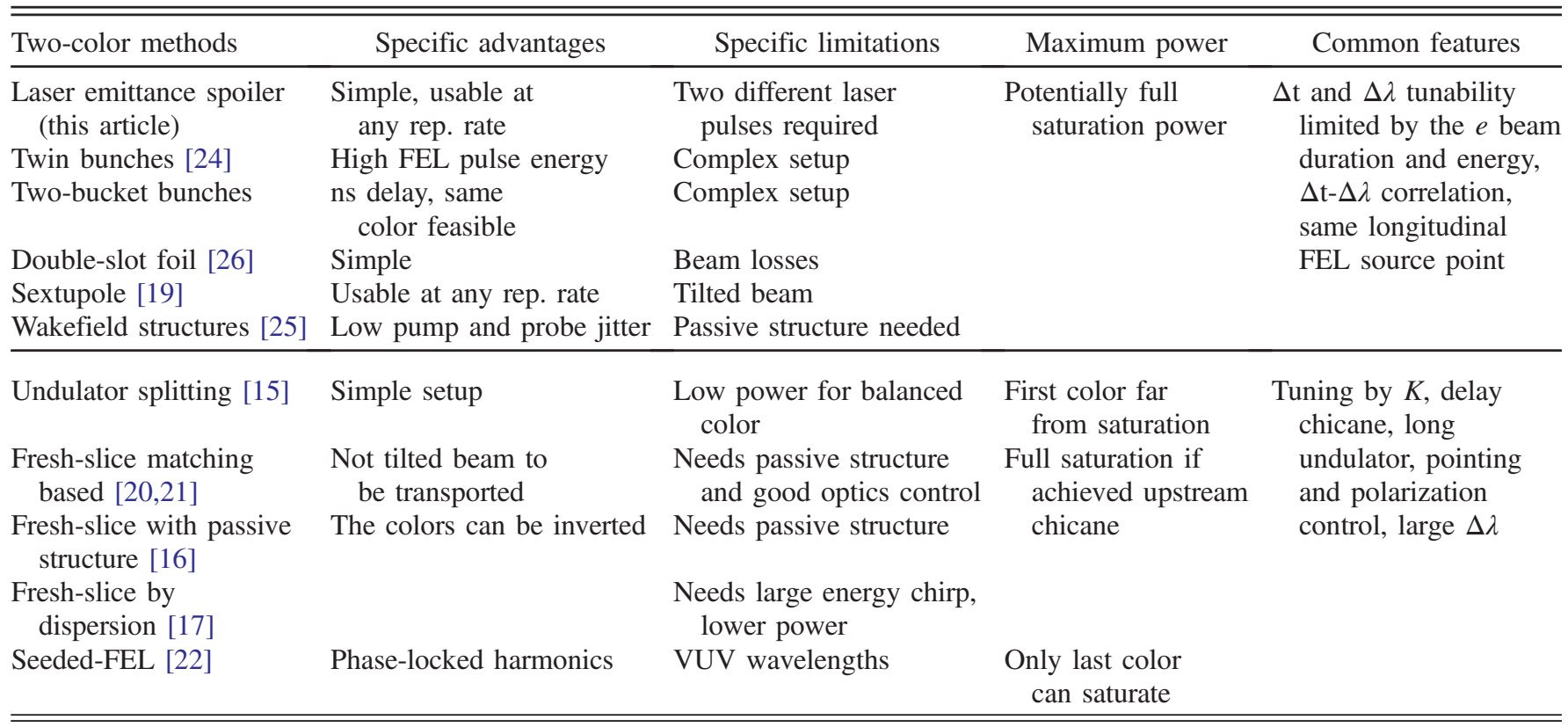

$\Delta t$, by changing the phase of the accelerator downstream of the compressors. The maximum $\Delta \lambda$ depends on the energy gain and the longitudinal wakefields. At SwissFEL, the maximum relative wavelength separation up to $2 \%$ was measured. Another parameter such as the relative intensity of the two colors can be easily varied by unbalancing the beam current distribution through the amplitude of the $\mathrm{x}$-band cavity linearizer. The laser emittance spoiler presented here adds a free knob for the control of the duration, the spectral width and the intensity of the two-color pulses. Moreover by increasing the intensity of the laser emittance spoiler, the temporal and wavelength separation between the two color pulses can be easily expanded without modifications of the compression settings.

The main characteristics of the two-color FEL methods demonstrated in literature are summarized in Table I. The methods based on electron beam manipulation are listed on the top rows, while the undulator-based two-color schemes are reported in the lower part of the table. We make a qualitative comparison between the laser emittance spoiler and other two-color methods including the specific advantages and limitations, with emphasis on the wavelength and the temporal separation $\Delta \lambda$ and $\Delta t$ respectively. A quantitative comparison between the different methods is not always possible due to the different machine layouts and accessible photon energies.

We experimentally demonstrated the two-color FEL by laser emittance spoiler at the hard $\mathrm{x}$-ray branch of SwissFEL (Aramis) [6]. At SwissFEL, $300 \mathrm{MeV}$ electron bunches at $100 \mathrm{~Hz}$, with a charge of $200 \mathrm{pC}$ and peak currents of $20 \mathrm{~A}$ are produced in a rf photoinjector by illuminating a $\mathrm{Cs}_{2} \mathrm{Te}$ photocathode. The linear accelerator boosts the beam energy up to $3.15 \mathrm{GeV}$ for the soft $\mathrm{x}$-ray FEL (Athos) and to $5.8 \mathrm{GeV}$ for the Aramis FEL. Two bunch compressors at 0.3 and $2.1 \mathrm{GeV}$ reduce the bunch length to a few tens of fs. Transverse deflector structures are routinely used to measure the electron bunch longitudinal properties such as the current, the horizontal slice emittance and the beam tilt. The Aramis undulator consists of 13 planar variable-gap modules of $4 \mathrm{~m}$ length each and a magnetic period of $15 \mathrm{~mm}$. This line produces radiation over the photon energy range of 1-7 $\AA$. The FEL pulse energies and spectra are measured by a gas detector and a single-shot spectrometer, respectively [29].

In Table II, the main parameters of the PC and emittance spoiler lasers at the photocathode are reported. The two systems consist of an ultralow noise $\mathrm{Yb}$-based oscillator and a diode-pumped $\mathrm{Yb}: \mathrm{CaF}_{2}$ amplifier running at $100 \mathrm{~Hz}$ [30]. Each laser produces pulses with $3 \mathrm{~mJ}$ energy and of $500 \mathrm{fs}$ FWHM duration. The amplified pulse is frequencyquadrupled to $260 \mathrm{~nm}$ reaching energies up to $300 \mu \mathrm{J}$. Both laser oscillators are synchronized with respect to the SwissFEL reference with a jitter of $11 \mathrm{fs}$ rms integrated

TABLE II. Parameters of the two laser pulses.

\begin{tabular}{lcc}
\hline \hline & $\begin{array}{c}\text { Nominal } \\
\text { PC laser }\end{array}$ & $\begin{array}{c}\text { Emittance } \\
\text { spoiler laser }\end{array}$ \\
\hline Wavelength (nm) & 260 & 260 \\
Duration FWHM (ps) & 6.6 & 0.95 \\
Diameter (mm) & 0.6 & 1.2 \\
Laser energy (nJ) & 130 & $7-22$ \\
Generated charge (pC) & 200 & $11-34$ \\
\hline \hline
\end{tabular}


from $2 \mathrm{mHz}$ to $100 \mathrm{~Hz}$. The relative time jitter between the two lasers at the photocathode is below $20 \mathrm{fs}$ rms. The two UV pulses are independently Fourier filtered in a glass capillary and are stretched in time with a grating pair. The stretcher is bypassed for the spoiler laser to keep the pulse duration short. To produce a top hat distribution on the photocathode, the beams are apertured and passed through an imaging transport. The spoiler pulse could also be derived from the same laser before the UV stretcher, but for the present study we used two independent systems. The laser spoiler can be activated on shot-to-shot basis, moreover it can be delayed and controlled in pulse energy and beam size. Space charge and electron beam emittance can be regulated via the intensity and the beam size of the emittance spoiler. The emittance spoiler and the photocathode lasers temporal profiles are measured with an optical cross-correlator and are reported in Fig. 1(b) and 1(c) respectively. As previously mentioned, the laser spoiler intensity can be tuned in order to locally exceed the emittance condition for the FEL amplification. At SwissFEL, a nominal emittance of $430 \mathrm{~nm}$ is required for the bunch to radiate at the shortest wavelength $(1 \AA)$ [6]. With a charge density peak, the emittance can be increased at the center of the bunch well beyond this limit preventing the FEL emission. In this way, only the external parts of the bunch with low emittance will contribute to the FEL and will produce a two-color spectrum.

In order to understand the underlying physics and define the operating parameters, we measured the electron beam slice emittance at the injector and upstream of the undulator line as function of the laser emittance spoiler intensity. We found experimentally that when the spoiler generates a charge of $19 \mathrm{pC}$ the emittance increases sufficiently to completely prevent the lasing of the corresponding part of the bunch and determines two distinct FEL emission peaks. At lower spoiler intensity the FEL emission is reduced at the two laser overlap but not completely suppressed. By increasing the spoiler charge above $19 \mathrm{pC}$, the emittance peak grows and a larger part of the beam exceeds the emittance limit resulting in the generation of a two-color FEL with narrower spectral width and larger wavelength separation.

Figure 2 shows the measured slice emittance as a function of the charge generated by the spoiler, which is reported in the legend of each plot. The dashed lines at -150 and 150 fs delimit visually the window where the overlap between the two lasers occurs at the photocathode. If the emittance spoiler laser is off, the slice emittance stays constant in the central part of the bunch with a value of $0.32 \mu \mathrm{m}$, well below the emittance limit for the FEL emission mentioned before. Already when the spoiler produces $11.5 \mathrm{pC}$, a peak in the emittance appears and the FEL amplification for the corresponding slice is reduced but not completely suppressed. At a generated spoiler charge larger than $15 \mathrm{pC}$, which corresponds to a slice emittance of $0.75 \mu \mathrm{m}$, the FEL radiation emission for these slices is completely inhibited. Increasing further the
Emittance

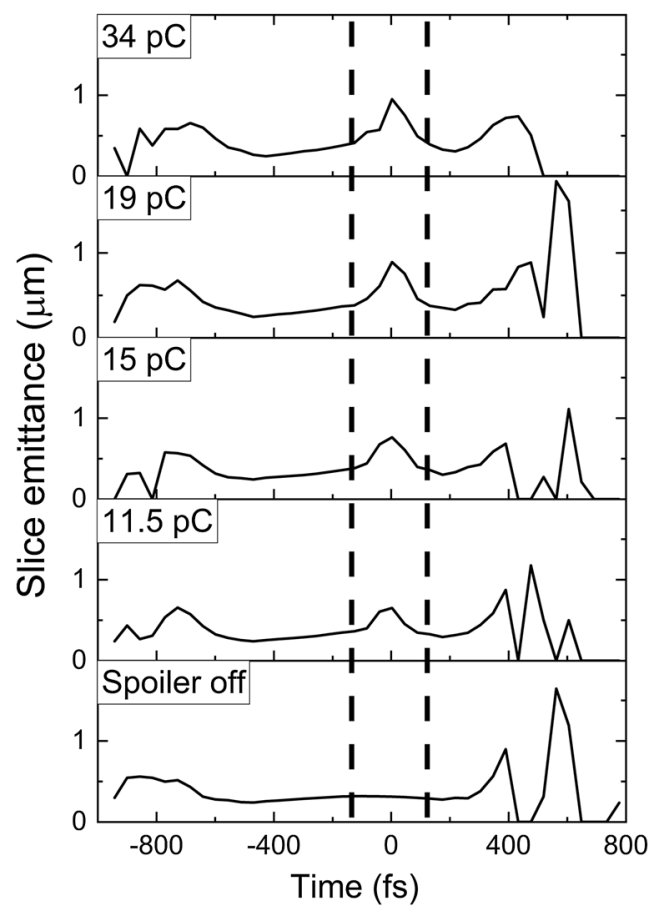

FIG. 2. Beam slice emittance measured at the injector for different laser spoiler pulse energies. In the legend of each graph, the charge generated by the laser spoiler is reported. The slice emittance stays constant along the bunch when the laser emittance spoiler is switched off (lower panel). As the laser emittance spoiler is activated and increased in intensity the emittance grows at the temporal overlap at time zero and is delimited by the two dashed lines at $-150 \mathrm{fs}$ and $150 \mathrm{fs}$.

spoiler charge, the emittance peak grows and a larger part of the beam exceeds the emittance limit resulting in the generation of a two-color FEL with narrower spectral width and larger wavelength separation. The emittance peak induced by the laser emittance spoiler is preserved along the accelerator. Figure 3 shows the slice emittance upstream of the undulator line for the laser emittance spoiler off (black line) and on (red line). For this measurement, the nominal photocathode laser is adjusted at the typical intensity for the generation of the $200 \mathrm{pC}$ bunch and the laser emittance spoiler was setup to produce a charge of $19 \mathrm{pC}$. The dashed lines delimit visually the temporal window where the overlap between the two lasers occurs at the photocathode. If the emittance spoiler laser is off, the slice emittance stays constant in the central part of the bunch below the emittance limit and the FEL emission mentioned occurs along the bunch. When the laser emittance spoiler is on, the FEL emission occurs over the two emittance minima and, due to the energy chirp in the electron beam, over two colors.

In the following, we present the experimental demonstration and characterization of the two-color FEL radiation. As aforementioned, the switch between the standard single and the two-color FEL can be done on a 


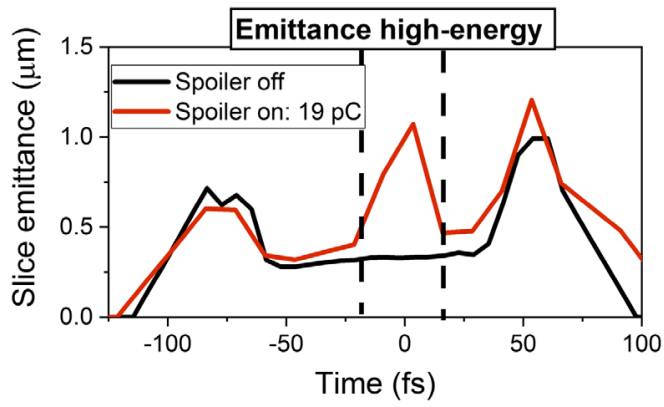

FIG. 3. Slice emittance measured at the end of the linear accelerator for laser emittance spoiler off (black curve) and when it produces $19 \mathrm{pC}$ (red curve).

shot-to-shot basis by enabling/disabling the emittance spoiler laser. The temporal structure of the two-color FEL can be estimated by the slice emittance of Fig. 3 . The FEL emission occurs in two few tens of femtosecond pulses separated by about $40 \mathrm{fs}$. One remarkable feature of the generation of the two-color FEL by laser emittance spoiler, is its excellent spectral shape stability. Figure 4 shows a set of 6000 consecutive SwissFEL spectra recorded at a photon energy of $12 \mathrm{keV}$. The statistical scattering of the spectral intensity for each photon energy is represented with the light purple area. The results clearly demonstrates that a stable wavelength two-color FEL with well-separated photon peaks can be reached for all the shots. The photon energy offset between the two colors and the peak wavelength remain stable. The stability of the spectral shape is an appealing feature for pump-probe experiments using two x-ray wavelenghs. Additionally, the present scheme allows for an easy reshaping of the FEL spectra. By changing the relative delay $\tau$ between the two laser pulses, it is possible to control the relative intensity, the spectral width and the duration of the two individual colors. Figure 5(a) shows the FEL spectra averaged over

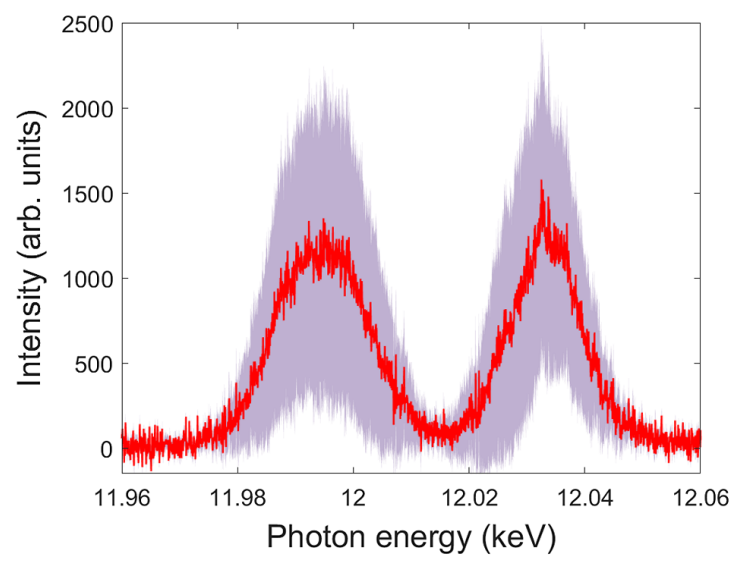

FIG. 4. Two-color FEL spectra. The red curve shows the average intensity and the light-purple area displays the statistical scattering recorded over 6000 consecutive spectra. (a)

FEL SPECTRA

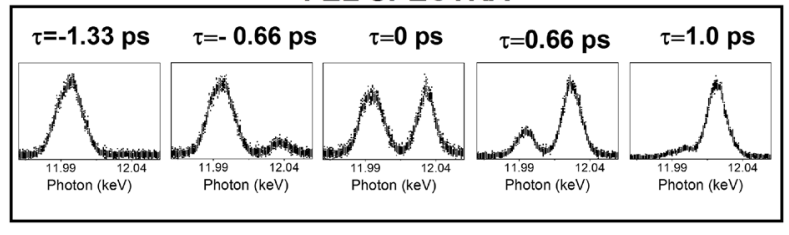

(b)

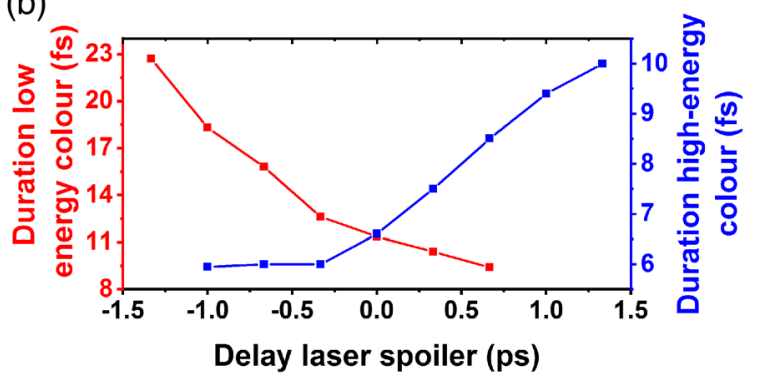

(c)

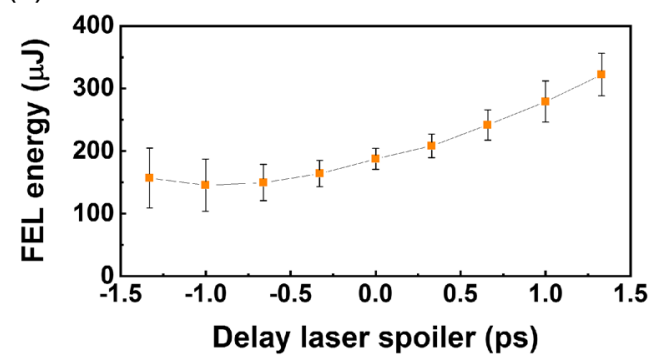

FIG. 5. (a) The two-color FEL spectra for different delays of the laser emittance spoiler, $\tau$, indicated above each graph. (b) The FWHM duration of the individual color pulses as a function of $\tau$ is retrieved through statistical analysis of the spectra. The red and the blue curves refer to the pulse duration for the longer and shorter wavelength color. (c) the corresponding average and standard deviation of the total FEL energy.

6000 pulses obtained by changing the relative delay between the two laser pulses. On top of each graph, the spoiler pulse delay is reported. When the emittance spoiler pulse overlaps with the centroid of the photocathode laser pulse, corresponding to $\tau=0$, the two FEL colors generated are balanced in intensity due to the uniform lasing along the bunch. The durations and the relative amplitude of the two pulses at $\tau=0 \mathrm{fs}$, are consistent with the slice emittance profile reported in Fig. 3. For $\tau= \pm 0.66 \mathrm{ps}$ the spectrum can be unbalanced toward the high or low energy color. For larger delay one of the colors is strongly reduced. With delays of the spoiler pulse larger than 1.33 ps the single spectral line FEL is recovered. The duration of the individual color is also controlled by the relative spectral content which is a function of the emittance spoiler pulse delay and intensity. The calculated FWHM pulse durations for the longer and shorter wavelength color as a function of the spoiler delay $\tau$ are reported in Fig. 5(b). These values are retrieved by fitting the second order spectral correlation function with the model derived in [31]. The spectra are numerically filtered taking into account the most relevant 
machine parameters which are recorded synchronously. As shown in the figure, adjusting the spoiler delay, the duration of the two FEL colors is significantly unbalanced and can be reduced significantly with minimum values of 6 and 9 fs FWHM for the long and short wavelength pulses. Figure 5(c) displays the averaged FEL pulse energy and the energy stability as a function of the delay of the laser spoiler. It is worth noting, that no special tuning of the machine is required to obtain the pulse energy in the two symmetric pulses of $190 \mu \mathrm{J}$, corresponding to $56 \%$ of the energy measured for standard single $\mathrm{x}$-ray color. In this condition, the standard deviation of the energy is $17 \mu \mathrm{J}$ $( \pm 9 \%)$, which is similar to the standard FEL energy stability confirming the excellent robustness of the presented scheme. The FEL energy increases for positive delays of the laser spoiler until reaching an energy of $320 \pm$ $34 \mu \mathrm{J}$ comparable with the pulse energy for normal SASE FEL operation. Delays of the spoiler toward lower values produce a reduction of the energy indicating that the electron bunch radiates more in the tail. This shows a potential application of the laser emittance spoiler as diagnostic to reveal which part of the electron beam contributes to the FEL process.

In conclusion, we present a simple and robust method to generate a two-color hard x-ray FEL. This novel approach relies on a laser emittance spoiler overlapped with the nominal photocathode laser. It enables the generation of two-color FEL pulses with high energy and spectral stability (energy $56 \%$ of the nominal FEL and comparable stability) with negligible interference to the machine setup. The accessible range for the wavelengths, wavelength separation and temporal delay of the two pulses is set by the electron bunch energy, chirp and duration of the single color FEL. the presented scheme allows shot-to-shot switching between single and two-color modes of operation which can be used for sophisticated lock-in detection schemes for pump-probe experiments. It does not contribute to additional charge losses along the machine and is therefore suitable for operation with the next generation high-repetition rate, high average power x-ray lasers. The reliability of the method and the ease of the setup responds to the demand of the FEL scientific community for lower risk two-color configurations to be used in advanced $\mathrm{x}$-ray pump, x-ray probe experiments. We are indebted to A. Cavalieri, G. Knopp and C. Beard for the valuable discussions. We thank all the SwissFEL technical groups for their support.

[1] P. Emma et al., First lasing and operation of an ångstromwavelength free-electron laser, Nat. Photonics 4, 641 (2010).

[2] T. Ishikawa et al., A compact x-ray free-electron laser emitting in the sub-ångström region, Nat. Photonics 6, 540 (2012).
[3] E. Allaria et al., Highly coherent and stable pulses from the fermi seeded free-electron laser in the extreme ultraviolet, Nat. Photonics 6, 699 (2012).

[4] W. Ackermann et al., Operation of a free-electron laser from the extreme ultraviolet to the water window, Nat. Photonics 1, 336 (2007).

[5] H.-S. Kang et al., Hard x-ray free-electron laser with femtosecond-scale timing jitter, Nat. Photonics 11, 708 (2017).

[6] C. J. Milne et al., Swissfel: The swiss x-ray free electron laser, Appl. Sci. 7, 720 (2017).

[7] C. Bostedt, S. Boutet, D. M. Fritz, Z. Huang, H. J. Lee, H. T. Lemke, A. Robert, W. F. Schlotter, J. J. Turner, and G. J. Williams, Linac coherent light source: The first five years, Rev. Mod. Phys. 88, 015007 (2016).

[8] P. M. Kraus, M. Zürch, S. K. Cushing, D. M. Neumark, and S.R. Leone, The ultrafast X-ray spectroscopic revolution in chemical dynamics, Nat. Rev. Chem. 2, 82 (2018).

[9] S. Schulz, I. Grguraš, C. Behrens, H. Bromberger, J. T. Costello, M. K. Czwalinna, M. Felber, M. C. Hoffmann, M. Ilchen, H. Y. Liu, T. Mazza, M. Meyer, S. Pfeiffer, P. Prędki, S. Schefer, C. Schmidt, U. Wegner, H. Schlarb, and A. L. Cavalieri, Femtosecond all-optical synchronization of an x-ray free-electron laser, Nat. Commun. 6, 5938 (2015).

[10] S. Serkez, W. Decking, L. Froehlich et al., Opportunities for two-color experiments in the soft x-ray regime at the european XFEL, Appl. Sci. 10, 2728 (2020).

[11] R. N. Coffee, J. P. Cryan, J. Duris, W. Helml, S. Li, and A. Marinelli, Development of ultrafast capabilities for X-ray free-electron lasers at the linac coherent light source, Phil. Trans. R. Soc. A 377, 20180386 (2019).

[12] F. Tissandier, J. Gautier, J.-P. Goddet, A. Kabacinski, S. Sebban, J. Nejdl, M. Kozlová, and G. Maynard, Two-Color Soft x-Ray Lasing in a High-Density Nickel-Like Krypton Plasma, Phys. Rev. Lett. 124, 133902 (2020).

[13] F. Bencivenga et al., Nanoscale transient gratings excited and probed by extreme ultraviolet femtosecond pulses, Sci. Adv. 5, eaaw5805 (2019).

[14] A. A. Lutman, R. Coffee, Y. Ding, Z. Huang, J. Krzywinski, T. Maxwell, M. Messerschmidt, and H.-D. Nuhn, Experimental Demonstration of Femtosecond TwoColor x-Ray Free-Electron Lasers, Phys. Rev. Lett. 110, 134801 (2013).

[15] T. Hara, Y. Inubushi, T. Katayama, T. Sato, H. Tanaka, T. Tanaka, T. Togashi, K. Togawa, K. Tono, M. Yabashi, and T. Ishikawa, Two-colour hard $\mathrm{x}$-ray free-electron laser with wide tunability, Nat. Commun. 4, 2919 (2013).

[16] A. A. Lutman, T. J. Maxwell, J. P. MacArthur, M. W. Guetg, N. Berrah, R. N. Coffee, Y. Ding, Z. Huang, A. Marinelli, S. Moeller, and J.C. U. Zemella, Fresh-slice multicolour X-ray free-electron lasers, Nat. Photonics 10, 745 (2016).

[17] M. W. Guetg, A. A. Lutman, Y. Ding, T. J. Maxwell, and Z. Huang, Dispersion-Based Fresh-Slice Scheme for FreeElectron Lasers, Phys. Rev. Lett. 120, 264802 (2018).

[18] S. Reiche and E. Prat, Two-color operation of a freeelectron laser with a tilted beam, J. Synchrotron Radiat. 23, 869 (2016). 
[19] P. Dijkstal, A. Malyzhenkov, S. Reiche, and E. Prat, Demonstration of two-color X-ray free-electron laser pulses with a sextupole magnet, Phys. Rev. Accel. Beams 23, 030703 (2020).

[20] W. Qin, Y. Ding, A. A. Lutman, and Y.-C. Chao, Matchingbased fresh-slice method for generating two-color $\mathrm{X}$-ray free-electron lasers, Phys. Rev. Accel. Beams 20, 090701 (2017).

[21] Y.-C. Chao, W. Qin, Y. Ding, A. A. Lutman, and T. Maxwell, Control of the Lasing Slice by Transverse Mismatch in an x-Ray Free-Electron Laser, Phys. Rev. Lett. 121, 064802 (2018).

[22] E. Ferrari et al., Widely tunable two-colour seeded freeelectron laser source for resonant-pump resonant-probe magnetic scattering, Nat. Commun. 7, 10343 (2016).

[23] C. Ronsivalle et al., Large-bandwidth two-color freeelectron laser driven by a comb-like electron beam, New J. Phys. 16, 033018 (2014).

[24] A. Marinelli, D. Ratner, A. A. Lutman, J. Turner, J. Welch, F. J. Decker, H. Loos, C. Behrens, S. Gilevich, A. A. Miahnahri, S. Vetter, T. J. Maxwell, Y. Ding, R. Coffee, S. Wakatsuki, and Z. Huang, High-intensity double-pulse X-ray free-electron laser, Nat. Commun. 6, 6369 (2015).

[25] S. Bettoni, E. Prat, and S. Reiche, Two-color beam generation based on wakefield excitation, Phys. Rev. Accel. Beams 19, 050702 (2016).

[26] A. S. Hernández, E. Prat, and S. Reiche, Generation of two-color X-ray free-electron-laser pulses from a beam with a large energy chirp and a slotted foil, Phys. Rev. Accel. Beams 22, 030702 (2019).

[27] A. Marinelli, R. Coffee, S. Vetter, P. Hering, G. N. West, S. Gilevich, A. A. Lutman, S. Li, T. Maxwell, J. Galayda, A. Fry, and Z. Huang, Optical Shaping of x-Ray Free-Electron Lasers, Phys. Rev. Lett. 116, 254801 2016).

[28] K.-J. Kim, Brightness, coherence and propagation characteristics of synchrotron radiation, Nucl. Instrum. Methods Phys. Res., Sect. A 246, 71 (1986).

[29] P. Juranić, J. Rehanek, C. A. Arrell, C. Pradervand, R. Ischebeck, C. Erny, P. Heimgartner, I. Gorgisyan, V. Thominet, K. Tiedtke, A. Sorokin, R. Follath, M. Makita, G. Seniutinas, C. David, C. J. Milne, H. Lemke, M. Radovic, C. P. Hauri, and L. Patthey, SwissFEL Aramis beamline photon diagnostics, J. Synchrotron Radiat. 25, 1238 (2018).

[30] A. Trisorio, M. Divall, C. Vicario, C. P. Hauri, and A. Courjaud, New concept for the SwissFEL gun laser, in Proceedings of FEL2013, New York, NY, USA (JACoW Publishing, New York, 2013), paper TUPSO88, pp. 442-446, http://www.jacow.org/FEL2013/papers/ tupso88.pdf.

[31] A. A. Lutman, Y. Ding, Y. Feng, Z. Huang, M. Messerschmidt, J. Wu, and J. Krzywinski, Femtosecond $\mathrm{X}$-ray free electron laser pulse duration measurement from spectral correlation function, Phys. Rev. ST Accel. Beams 15, 030705 (2012). 